\title{
SOME RESULTS CONCERNING HYPERBOLIC MANIFOLDS
}

\author{
PETER KIERNAN
}

\begin{abstract}
A complex manifold is (complete) hyperbolic if the Kobayashi pseudo-distance is a (complete) distance. In this note, it is shown that a fibre bundle is (complete) hyperbolic if both the base and fibre are (complete) hyperbolic. Two examples are also given. The first shows that the completion of a hyperbolic manifold is not necessarily locally compact. The second shows that one generalization of the big Picard theorem is false.
\end{abstract}

In this note, we give an example of a hyperbolic manifold $M$ whose completion $M^{*}$ is not locally compact. It is also shown that a fibre bundle $E$ is (complete) hyperbolic if both the base and fibre are (complete) hyperbolic.

We begin by recalling the definition of the Kobayashi pseudodistance $d_{M}$ associated to the complex manifold $M$. Let $p$ and $q$ be points in $M$. By a chain $\alpha$ from $p$ to $q$, we mean a sequence $p=p_{0}$, $p_{1}, \cdots, p_{k}=q$ of points in $M$, points $a_{1}, \cdots, a_{k}$ in the unit disk $D=\{z \in C|| z \mid<1\}$ and holomorphic maps $f_{1}, \cdots, f_{k}$ of $D$ into $M$ with $f_{i}(0)=p_{i-1}$ and $f_{i}\left(a_{i}\right)=p_{i}$. The length $|\alpha|$ of $\alpha$ is defined by

$$
|\alpha|=\sum_{i=1}^{k} d\left(0, a_{i}\right)=\sum_{i=1}^{k} \log \frac{1+\left|a_{i}\right|}{1-\left|a_{i}\right|}
$$

where $d$ is the Poincaré-Bergman distance on $D$. It is given by the metric $d s^{2}=\left(1 /\left(1-|z|^{2}\right)^{2}\right) d z d \bar{z}$. We set $d_{M}(p, q)=\inf _{\alpha \in A}|\alpha|$, where $A$ is the set of all chains from $p$ to $q$. It is easy to see that $d_{M}$ is a pseudodistance on $M$. If $d_{M}$ is an actual distance, we say that $M$ is hyperbolic. $M$ is called complete hyperbolic if $d_{M}$ is a complete metric, i.e., if all Cauchy sequences converge.

It follows immediately from the definition of $d_{M}$ and $d_{N}$, that if $f: M \rightarrow N$ is holomorphic and $p, q \in M$, then $d_{N}(f(p), f(q)) \leqq d_{M}(p, q)$. The classical Schwarz-Pick lemma implies that the Kobayashi distance $d_{D}$ on the unit disk $D$ is the same as the Poincaré-Bergman distance $d$.

The following example is modeled after one due to D. B. A. Epstein of a Riemannian manifold whose completion is not locally compact. Let

Received by the editors December 8, 1969.

AMS Subject Classifications. Primary 3225, 3260, 5340, 5399.

Key Words and Phrases. Hyperbolic, Kobayashi pseudo-distance, big Picard theorem, fibre bundle, completion, locally compact. 
$A=\{z=x+i y \in D$ where $y=0$, and $x=1 / n$ or $x \leqq 0\}$, $A_{t}=\{w=u+i v \in D$ where $u>t \geqq 0\}$ and $M=D \times D-A \times \bar{A}_{0}$. Since $M$ is an open set of $D \times D$, it is hyperbolic. Let $M^{*}$ denote its completion with respect to $d_{M}$.

Theorem 1. $M^{*}$ is not locally compact.

Proof. (a) Since the inclusion $i: M \rightarrow D \times D$ is distance decreasing and since $D \times D$ is complete, we can consider $M^{*}$ as being embedded in $D \times D$. Using $(z, w)$ where $z=x+i y$ and $w=u+i v$ as coordinates in $D \times D$, we shall show that the sequence $\left\{p_{n}=(0,-1 / n)\right\}$ is Cauchy with respect to $d_{M}$. This will show that $(0,0) \in M^{*}$.

Let $\epsilon>0$ be given. Choose an irrational number $r>0$ such that $z \in D_{r}=\{z \in D|| z \mid<r\}$ implies $d_{D}(z, 0)<\epsilon / 4$. If $m>1 / r$ and $n>1 / r$, then

$$
\begin{aligned}
d_{M}\left(p_{n}, p_{m}\right) \leqq & d_{M}\left(\left(0, \frac{-1}{n}\right),\left(\frac{r}{2}, \frac{-1}{n}\right)\right) \\
& +d_{M}\left(\left(\frac{r}{2}, \frac{-1}{n}\right),\left(\frac{r}{2}, \frac{-1}{m}\right)\right) \\
& +d_{M}\left(\left(\frac{r}{2}, \frac{-1}{m}\right),\left(0, \frac{-1}{m}\right)\right)<4 \frac{\epsilon}{4}=\epsilon .
\end{aligned}
$$

The last step uses the fact that holomorphic mappings are distance decreasing. Thus $\left\{p_{n}\right\}$ is a Cauchy sequence.

(b) Let $\left\{q_{n}\right\}$ be a Cauchy sequence in $M$. There exists $q_{0}=\left(z_{0}, w_{0}\right)$ $\in D \times D$ such that $q_{n} \rightarrow q_{0}$. Suppose $w_{0}=u_{0}+i v_{0}$ where $u_{0}>t>0$. Let $\pi: D \times D \rightarrow D$ be the projection onto the second factor and let $B_{s}$ be the ball of radius $s$ (with respect to $d_{D}$ ) centered at $\pi\left(q_{0}\right)$. Choose $s>0$ such that $B_{4 s} \subset A_{t}$ and choose $r>0$ such that $z \in D_{r}$ implies $d_{D}(z, 0)<s$.

We shall show that $\left\{q_{n}\right\}$ is Cauchy with respect to $d_{N}$ where $N=(D-A) \times A_{t}$. We can assume that $q_{n} \in \pi^{-1}\left(B_{s}\right)$ for all $n$. The choices above imply that in computing $d_{M}\left(q_{n}, q_{m}\right)$, we can use chains of the form $\alpha=\left\{f_{1}, \cdots, f_{k} ; a_{1}, \cdots, a_{k} ; p=x_{0}, \cdots, x_{k}=q_{m}\right\}$ where $x_{i} \in \pi^{-1}\left(B_{2 s}\right) \cap N, a_{i} \in D_{r / 2}$ and $f_{i}\left(D_{r}\right) \subset N$. Choose $c>0$ such that $a \in D_{r / 2}$ implies that $c d_{D}(0, a) \geqq d_{D_{r}}(0, a)$. Since

$$
c|\alpha|=c \sum_{i=1}^{k} d_{D}\left(0, a_{i}\right) \geqq \sum_{i=1}^{k} d_{D_{r}}\left(0, a_{i}\right) \geqq d_{N}\left(q_{n}, q_{m}\right),
$$

we have $c d_{M}\left(q_{n}, q_{m}\right) \geqq d_{N}\left(q_{n}, q_{m}\right)$. Thus $\left\{q_{n}\right\}$ is Cauchy with respect 
to $d_{N}$. But $N$ is complete, since it is the product of two complete hyperbolic spaces. This shows that $q_{0} \in N \subset M$.

(c) Let $U=\left\{(z, w) \in M^{*}|| z \mid<r\right.$ and $|w|<r$ for some $\left.r>0\right\}$ and let $B=\left\{\left(3 / 2^{n}, r / 2\right) \mid 3 / 2^{n}<r\right\}$. Then $B$ is an infinite set contained in $U$ and the argument in (b) shows that $B$ has no accumulation point in $M^{*}$. Thus $M^{*}$ is not locally compact. q.e.d.

We shall now consider a complex analytic fibre bundle $\pi: E \rightarrow M$ with fibre $F$.

THEOREM 2. E is (complete) hyperbolic if both $M$ and $F$ are (complete) hyperbolic.

Proof. (a) Assume that $M$ and $F$ are hyperbolic. Let $p, q \in E$. If $\pi(p) \neq \pi(q)$, then $d_{E}(p, q)>0$. If $\pi(p)=\pi(q)$, choose a neighborhood $U$ of $p_{0}=\pi(p)$ with $\pi^{-1}(U)=U \times F$. Let $B_{s}$ be the ball in $M$ centered at $p_{0}$ and of radius $s$ with respect to $d_{M}$. Choose $s>0$ and $r>0$ such that $B_{2 s} \subset U$ and $z \in D_{r}$ implies $d_{D}(z, 0)<s$. Thus, if $f: D \rightarrow E$ is holomor phic and $f(0) \in \pi^{-1}\left(B_{s}\right)$, then $f\left(D_{r}\right) \subset U \times F$. Choose $c>0$ such that $a \in D_{r / 2}$ implies $d_{D}(a, 0) \geqq c d_{D_{r}}(a, 0)$. Let $\alpha=\left\{f_{1}, \cdots, f_{k} ; a_{1}, \cdots, a_{k}\right.$; $\left.x_{0}=p, x_{1}, \cdots, x_{k}=q\right\}$ be given a chain from $p$ to $q$ with $x_{i} \in \pi^{-1}\left(B_{s}\right)$ and $a_{i} \in D_{r / 2}$. Then

$$
\begin{aligned}
|\alpha| & =\sum_{i=1}^{k} d_{D}\left(a_{i}, 0\right) \geqq c \sum_{i=1}^{k} d_{D_{r}}\left(a_{i}, 0\right) \\
& \geqq c \sum_{i=1}^{k} d_{F}\left(\phi\left(x_{i}\right), \phi\left(x_{i-1}\right)\right) \geqq c d_{F}(p, q)>0 \quad \text { if } p \neq q
\end{aligned}
$$

where $\phi: U \times F \rightarrow F$ is the projection. Thus $E$ is hyperbolic.

(b) Assume that $M$ and $F$ are complete hyperbolic and let $\left\{p_{n}\right\}$ be a Cauchy sequence in $E$. Then $\left\{\pi\left(p_{n}\right)\right\}$ is Cauchy in $M$ and therefore $\pi\left(p_{n}\right) \rightarrow p_{0}$ for some $p_{0} \in M$. Using the notation as in (a), we have $p_{n} \in \pi^{-1}\left(B_{s}\right)$ for $n$ large. Let $\alpha$ be a chain as above with $p$ and $q$ replaced by $p_{n}$ and $p_{m}$. Then

$$
\begin{aligned}
\frac{1}{c}|\alpha| & =\frac{1}{c} \sum_{i=1}^{k} d_{D}\left(a_{i}, 0\right) \geqq \sum_{i=1}^{k} d_{D_{r}}\left(a_{i}, 0\right) \\
& \geqq \sum_{i=1}^{k} d_{F}\left(\phi\left(x_{i}\right), \phi\left(x_{i-1}\right)\right) \geqq d_{F}\left(\phi\left(p_{n}\right), \phi\left(p_{m}\right)\right) .
\end{aligned}
$$

This shows that $\left\{\phi\left(p_{n}\right)\right\}$ is Cauchy in $F$ and therefore $\phi\left(p_{n}\right) \rightarrow p$ for some $p \in F$. Clearly $p_{n} \rightarrow\left(p_{0}, p\right)$. Thus $E$ is complete. q.e.d.

Special cases of the previous theorem were proved earlier by Kobayashi and Hirschfelder. 
THEOREM 3. Let $\pi: E \rightarrow M$ be a complex analytic vector bundle over a hyperbolic manifold $M$. Then there exists a neighborhood $N$ of the zero section such that $N$ is hyperbolic.

Proof. Let $h$ be a hermitian metric in $E$ and let

$$
N=\{v \in E \mid h(v, v)<1\} .
$$

If $p, q \in N$ and $\pi(p) \neq \pi(q)$ then $d_{N}(p, q)>0$. If $\pi(p)=\pi(q)$, choose a relatively compact neighborhood $U$ of $p_{0}=\pi(p)$ with $\pi^{-1}(U)=U \times C^{n}$. Let $B_{s}, s, r$, and $c$ be as in the first part of the previous proof. If $f: D \rightarrow N$ is holomorphic and $\pi(f(0)) \in B_{s}$, then $f\left(D_{r}\right) \subset\left(U \times C^{n}\right) \cap N$. Without loss of generality, we can assume that $N \cap\left(U \times C^{n}\right) \subset U \times B^{n}$ where $B^{n}$ is the unit ball in $C^{n}$. Let $\phi: U \times B^{n} \rightarrow B^{n}$ be the projection and let $\alpha=\left\{f_{1}, \cdots, f_{k} ; a_{1}, \cdots, a_{k} ; x_{0}=p, x_{1}, \cdots, x_{k}=q\right\}$ be a chain from $p$ to $q$ with $x_{i} \in \pi^{-1}\left(B_{s}\right) \cap N$ and $a_{i} \in D_{r / 2}$. Then

$$
\begin{aligned}
|\alpha| & \geqq \sum_{i=1}^{k} d_{D}\left(a_{i}, 0\right) \geqq c \sum_{i=1}^{k} d_{D_{r}}\left(a_{i}, 0\right) \\
& \geqq c \sum_{i=1}^{k} d_{B^{n}}\left(\phi\left(x_{i}\right), \phi\left(x_{i-1}\right)\right) \geqq c d_{B^{n}}(p, q)>0 \quad \text { if } p \neq q .
\end{aligned}
$$

Thus $N$ is hyperbolic. q.e.d.

The big Picard theorem can be stated in the following way. Let $D^{*}=\{z \in C|0<| z \mid<1\}$ and let $M \subset P_{1}(C)$ be a hyperbolic open submanifold. Then any holomorphic map $f: D^{*} \rightarrow M$ can be extended to a holomorphic map $f: D \rightarrow P_{1}(C)$. In [3], M. Kwack showed that if $N$ is a compact hyperbolic manifold and $f: D^{*} \rightarrow N$ is holomorphic, then $f$ can be extended to the whole disk $D$. Kobayashi (see [2]) has proved the following. Let $M$ and $Y$ be complex manifolds with

(1) $M \subset Y$ and $\bar{M}$ compact,

(2) $M$ hyperbolic,

(3) given a point $p$ of $\bar{M}-M$ and a neighborhood $U$ of $p$ in $Y$, there exists a neighborhood $V$ of $p$ in $Y$ such that $\bar{V} \subset U$ and the distance between $M \cap(Y-U)$ and $M \cap V$ with respect to $d_{M}$ is positive. Then every holomorphic mapping $f$ from the punctured disk $D^{*}$ into $M$ can be extended to a holomorphic mapping from the disk $D$ into $Y$.

The following example indicates that the assumptions above are probably necessary and also shows that the big Picard theorem cannot be generalized to higher dimensions in the form given above.

The tangent bundle $T\left(D^{*}\right)$ of $D^{*}$ can be identified with $D^{*} \times C \subset C^{2} \subset P_{2}(C)$. Define a hermitian metric $h$ on $D^{*}$ by $d s^{2}$ $=\left(1 / 2 e^{2 /|z|}\right) d z d \bar{z}$ and let $M=\left\{v \in T\left(D^{*}\right) \mid h(v, v)<1\right\}$. The proof of 
Theorem 3 shows that $M$ is hyperbolic. (Using similar techniques, it can be shown that $M$ is complete hyperbolic.) Define $f: D^{*} \rightarrow M$ $C P_{2}(C)$ by $f(z)=\left(z, e^{1 / z}\right)$. It is easy to see that $f$ cannot be extended to a holomorphic map $f: D \rightarrow P_{2}(C)$.

\section{REFERENCES}

1. Shoshichi Kobayashi, Invariant distances on complex manifolds and holomorphic mappings, J. Math. Soc. Japan 19 (1967), 460-480.

2. - Hyperbolic manifolds and holomorphic mappings, Lecture Notes in Math., Springer, New York (to appear).

3. M. Kwack, Generalization of the big Picard theorem, Ann. of Math. (2) 90 (1969), 9-23.

University of California, Berkeley, California 94720 